\title{
Wavefront and Divergence of the Beamlet Prototype Laser
}

\author{
P. J. Wegner, M. A. Henesian, J. T. Salmon, \\ L. G. Seppala, T. L. Weiland, W. H. Williams, \\ and B. M. Van Wonterghem
}

This paper was prepared for submittal to the Third Annual International Conference on Solid State Lasers for Application (SSLA)

to Inertial Confinement Fusion (ICF)

Monterey, California

June 7-12, 1998

October 30, 1998

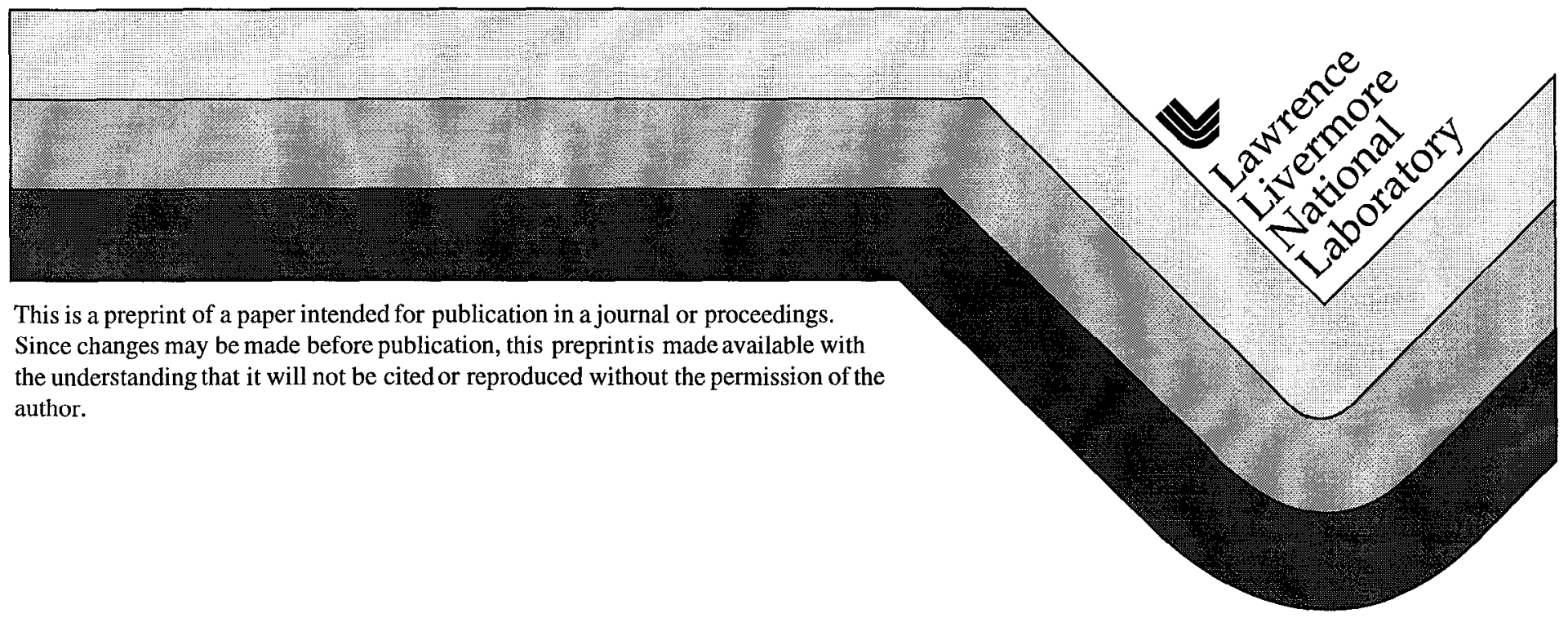




\section{DISCLAIMER}

This document was prepared as an account of work sponsored by an agency of the United States Government. Neither the United States Government nor the University of California nor any of their employees, makes any warranty, express or implied, or assumes any legal liability or responsibility for the accuracy, completeness, or usefulness of any information, apparatus, product, or process disclosed, or represents that its use would not infringe privately owned rights. Reference herein to any specific commercial product, process, or service by trade name, trademark, manufacturer, or otherwise, does not necessarily constitute or imply its endorsement, recommendation, or favoring by the United States Government or the University of California. The views and opinions of authors expressed herein do not necessarily state or reflect those of the United States Guverrument or the University of California, and shall not be used for advertising or product endorsement purposes. 


\author{
Wavefront and divergence of the Beamlet prototype laser \\ P.J. Wegner, M.A. Henesian, J.T. Salmon, L.G. Seppala, T.L. Weiland, \\ W.H. Williams and B.M. Van Wonterghem \\ University of California, Lawrence Livermore National Laboratory \\ P.O. Box 808 , L-479, Livermore, CA 94550
}

\begin{abstract}
We have measured the wavefront and the divergence of the Beamlet prototype laser under a variety of conditions. Emphasis of the tests was on quantifying best attainable divergence in the angular regime below $30 \mu \mathrm{rad}$ to benchmark propagation models that are used to set wavefront gradient specifications for NIF optical components. Performance with and without active wavefront correction was monitored with radial shearing interferometers that measured near-field wavefront at the input and output of the main amplifier with a spatial resolution of $1 \mathrm{~cm}$, and cameras which measured the corresponding intensity distributions in the far field with an angular resolution of $0.3 \mu \mathrm{rad}$. Details of the measurements are discussed and related to NIF focal spot requirements and optics specifications.
\end{abstract}

Keywords: interferometry, adaptive optics, solid-state lasers, ICF

\title{
1. INTRODUCTION
}

Certain target requirements for the National Ignition Facility (NIF) call for the delivery of $500 \mathrm{TW}$ of $0.351-\mu \mathrm{m}$ $(3 \omega)$ radiation inside a $250-\mu \mathrm{m}$ diameter focal spot $[1,2]$. For the $7.7-\mathrm{m}$ focal-length lenses on the NIF target chamber this spot size corresponds to a half angle of $16 \mu \mathrm{rad}$, which sets a stringent upper limit for the divergence of the laser. There are several sources of divergence in the laser, primarily in the $1.053-\mu \mathrm{m}(1 \omega)$ section, that can significantly degrade the quality of the focal spot unless mitigated or otherwise controlled. These sources fall readily into four categories: thermally induced phase errors related to heat accumulation in the amplifiers, including gas turbulence effects [3,4]; prompt phase errors related to a rapid deformation of the amplifier slabs during pumping [5]; static phase errors related to the finishing, mounting and alignment of the optical components; and nonlinear phase errors associated with intensity-dependent ripple growth and whole-beam self focusing (B integral) that occur at high power [6-8]. At a given power level, minimum divergence and maximum laser brightness is achieved when the system is cold and thermally induced phase errors are absent. In this case, performance is primarily limited by the fraction of prompt and static phase errors that remain uncorrected by the wavefront control adaptive optic system.

Recent wavefront characterization experiments conducted on the Beamlet prototype laser [9] have played an important role in ensuring that the NIF will meet its focal spot requirements. As a result of this work we have quantified the beam quality at the injection point to the main amplifier; static phase errors in the main amplifier with the system cold; prompt pump-induced phase errors in the main amplifier; limits of phase error correction with the adaptive optics system; and the best attainable output beam divergence at both low and high power. Important applications of the data have been the validation of NIF propagation models, and the establishment of transmitted wavefront specifications for NIF optics. The next section contains a brief description of the Beamlet measurements, and summarizes the results for the different laser configurations tested. Results are discussed in Section 3. Specific details of the measurements are covered in the appendix.

\section{BEAMLET MEASUREMENTS}

The Beamlet laser, shown schematically in Figure 1, is equipped with an adaptive optics system (AOS) to compensate for wavefront aberrations in both the preamplifier and main amplifier $[10,11]$. The active component in the Beamlet AOS is a $7-\mathrm{cm}$ square deformable mirror (DFM) with 39 independent actuators; the number of actuators and their arrangement is similar in design to the 40-cm mirror that will be deployed in the main amplifier cavity on the NIF [12]. On Beamlet, the mirror resides at the output of the preamplifier and conditions the wavefront of the pulse before it is injected 


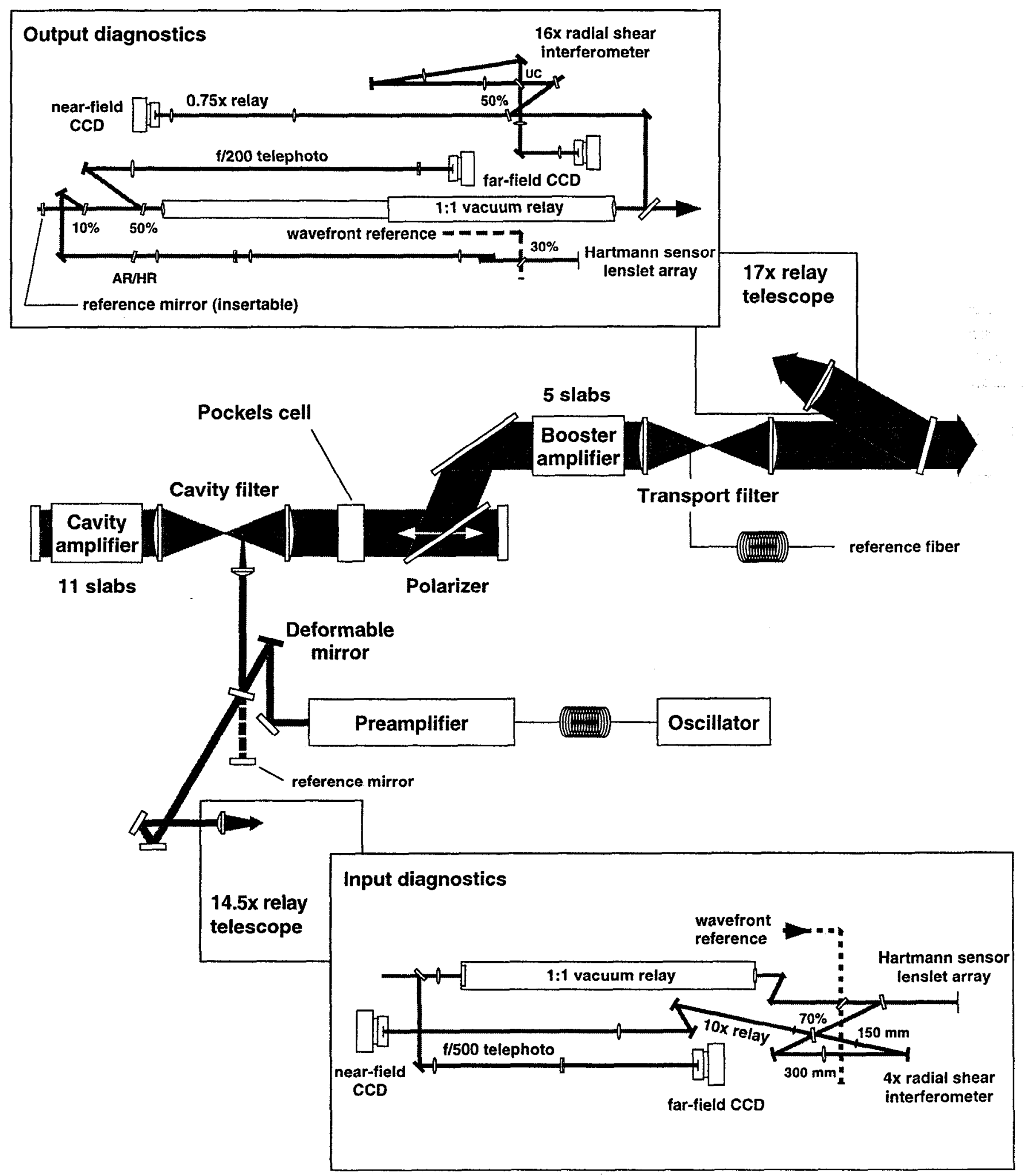

Figure 1. Schematic of the Beamlet laser system showing locations of the deformable mirror and relevant diagnostics. Beam sizes at the ouputs of the preamplifier and main amplifer are 5 and $34 \mathrm{~cm}$, respectively. 
into the main amplifier cavity. Wavefront data for closed-loop control of the mirror is provided by either of two 77-element Hartmann sensors located in diagnostic packages situated at the input and output of the main amplifier. Closed-loop control allows the figure of the mirror to be updated continuously $(\sim 1 \mathrm{~Hz})$ to maintain a predefined wavefront at the Hartmann sensor, which is typically specified to be either "flat" or a compensating figure determined from the wavefront error measured on a previous shot (termed "prefigure"). Additional diagnostics check the operation of the AOS and quantify beam quality. The primary tool that we have used for this task is the radial shear interferometer. A 4-x radial-shear interferometer in the input diagnostic package measures wavefront at the plane of the DFM. A 16-x device located in the output diagnostics measures wavefront at the output relay plane of the transport spatial filter, which is the location of the frequency converter. High-magnification far-field cameras in both packages measure the focal spot intensity distribution. A detailed description of the diagnostics and the methods used to extract wavefront from the radial shear data are presented in the appendix.

Measurements of wavefront and divergence at low power were obtained under a variety of conditions to quantify system performance and optimize the AOS in the absence of nonlinear phase retardation effects. Table 1 lists the eleven configurations of the laser that were tested: the preamplifier of the laser up to the plane of the DFM, and the full system up to the output of the transport spatial filter, for several configurations of the DFM (including a flat mirror in place of the DFM) and with the 5-cm rod in the preamplifier and 40-cm slabs in the main amplifier either static or pumped. All of the measurements were performed with the system cold, meaning that the minimum time interval between shots was 20 minutes for the preamplifier, and 14 hours for the main amplifier. Output power was held below 0.7 TW in 3 ns. Performance of the optimized system was also measured at high power as discussed in the next Section.

\begin{tabular}{|c|c|c|c|c|}
\hline Configuration & AOS wavefront* & preamp & main amp & Description \\
\hline \multirow[t]{2}{*}{ preamp, no DFM } & none & static & - & static front end \\
\hline & none & pumped & - & static front end plus pumped rod \\
\hline \multirow[t]{3}{*}{ preamp, w/ DFM } & flat at input & static & - & $\begin{array}{l}\text { residual error of mirror (correcting } \\
\text { static front end) }\end{array}$ \\
\hline & flat at input & pumped & - & mirror residual plus pumped rod \\
\hline & prefigured at output & pumped & - & front end shot condition \\
\hline system, no DFM & none & pumped & static & static system plus pumped rod \\
\hline \multirow[t]{5}{*}{ system, w/ DFM } & flat at input & pumped & static & routine system check \\
\hline & flat at output & pumped & static & $\begin{array}{l}\text { residual error of mirror (correcting } \\
\text { static system) plus pumped rod }\end{array}$ \\
\hline & flat at output & pumped & pumped & $\begin{array}{l}\text { residual error of mirror plus total } \\
\text { pump-induced error (rod plus } \\
\text { slabs) }\end{array}$ \\
\hline & prefigured at output & pumped & static & prefigured wavefront \\
\hline & prefigured at output & pumped & pumped & output shot condition \\
\hline
\end{tabular}

* closed-loop operation to maintain indicated wavefront at indicated Hartmann sensor until 1 second prior to shot

Table 1. Configuration summary of cold-system tests at low power

A summary of the low-power test resultsis given in Table 2, which lists values for several parameters that have been calculated from the measured wavefronts: peak-to-valley error, root-mean-squared (RMS) error, RMS gradient, Strehl ratio, divergence half angle ( $80 \%$ power) and peak far-field intensity. To calculate these quantities we used a simplified flattop model for the amplitude of the Beamlet beam that has the following characteristics: a beam size of $340 \mathrm{~mm}$ at the $1 \%$ level, beam corners of radius $r_{c}=45 \mathrm{~mm}$, and an edge roll off defined by a $10^{\text {th }}$ power super-gaussian $\exp \left[-\left(r / r_{0}\right)^{10}\right]$ with $r_{0}=$ $r_{c} /(\ln 100)^{1 / 10}$ and the origin $(r=0)$ located a distance $r_{c}$ in from the beam edge. The edge profile produced by this model is not exact, but is very similar to that which we measure on Beamlet. Sampling for the wavefront statistics (peak-to-valley error, RMS error, RMS gradient) was restricted to the area of the beam having amplitude greater than or equal to $50 \%$ of the peak, to avoid the edges of the beam where the signal level is low and the uncertainty in the measured wavefront is large. Strehl ratio was calculated from the following formula: 


\begin{tabular}{|c|c|c|c|c|c|c|c|c|c|c|c|c|}
\hline \multirow[b]{2}{*}{ Configuration } & \multirow[b]{2}{*}{ AOS wavefront } & \multicolumn{9}{|c|}{ Interferometry } & \multicolumn{2}{|c|}{ Far-field data } \\
\hline & & $\begin{array}{l}\text { pun } \\
\text { rod }\end{array}$ & $\begin{array}{l}\text { sped } \\
\text { slabs }\end{array}$ & $\#$ & $\begin{array}{l}\text { P-V error } \\
\text { waves }\end{array}$ & $\begin{array}{c}\text { RMS error } \\
\text { waves }\end{array}$ & $\begin{array}{l}\text { RMS grad* } \\
\text { waves/cm }\end{array}$ & $\begin{array}{l}\text { Strehl } \\
\text { ratio }\end{array}$ & $\begin{array}{c}80 \% 1 / 2 \\
\text { angle } \\
\mu \mathrm{rad}\end{array}$ & $\begin{array}{c}\text { peak } \\
\text { intensity } * *\end{array}$ & $\begin{array}{c}80 \% 1 / 2 \\
\text { angle } \\
\mu \mathrm{rad}\end{array}$ & $\begin{array}{c}\text { peak } \\
\text { intensity** }\end{array}$ \\
\hline \multirow[t]{2}{*}{ preamp, no DFM } & none & - & - & 1 & 0.57 & 0.10 & 0.03 & 0.69 & 2.97 & 6.97 & - & - \\
\hline & none & $x$ & - & 2 & $0.52 / 0.06$ & $0.10 / 0.00$ & $0.03 / 0.00$ & $0.70 / 0.02$ & $3.57 / 0.59$ & $7.22 / 0.30$ & $4.34 / 0.44$ & $5.84 / 0.26$ \\
\hline \multirow[t]{3}{*}{ preamp, w/DFM } & flat at input & - & - & 2 & $0.32 / 0.05$ & $0.06 / 0.01$ & $0.03 / 0.00$ & $0.87 / 0.05$ & $2.76 / 0.19$ & $8.43 / 0.34$ & - & - \\
\hline & flat at input & $\mathrm{x}$ & - & 3 & $0.51 / 0.06$ & $0.08 / 0.01$ & $0.03 / 0.00$ & $0.78 / 0.04$ & $4.59 / 0.46$ & $7.71 / 0.22$ & $4.26 / 0.34$ & $7.88 / 0.12$ \\
\hline & prefigured at output & $\mathrm{x}$ & - & 4 & $2.78 / 0.26$ & $0.63 / 0.04$ & $0.16 / 0.01$ & $0.00 / 0.00$ & $20.5 / 2.11$ & $0.42 / 0.14$ & - & - \\
\hline system, no DFM & none & $\mathrm{x}$ & - & 2 & $1.63 / 0.01$ & $0.33 / 0.00$ & $0.13 / 0.00$ & $0.01 / 0.00$ & $15.6 / 1.03$ & $1.07 / 0.27$ & $15.95 / 0.83$ & $1.20 / 0.23$ \\
\hline \multirow[t]{5}{*}{ system, w/ DFM } & flat at input & $\mathrm{x}$ & - & 3 & $1.90 / 0.16$ & $0.41 / 0.06$ & $0.14 / 0.03$ & $0.01 / 0.01$ & $14.1 / 0.41$ & $0.94 / 0.13$ & $14.55 / 1.84$ & $0.97 / 0.13$ \\
\hline & flat at output & $\mathrm{x}$ & - & 4 & $1.05 / 0.15$ & $0.19 / 0.02$ & $0.10 / 0.00$ & $0.23 / 0.09$ & $10.9 / 1.82$ & $3.32 / 0.86$ & $10.45 / 0.57$ & $3.22 / 0.69$ \\
\hline & flat at output & $\mathrm{x}$ & $x$ & 4 & $3.02 / 0.06$ & $0.56 / 0.01$ & $0.18 / 0.00$ & $0.05 / 0.01$ & $26.5 / 0.48$ & $0.52 / 0.09$ & $26.49 / 1.65$ & $0.81 / 0.16$ \\
\hline & prefigured at output & $x$ & - & 3 & $2.23 / 0.20$ & $0.40 / 0.01$ & $0.16 / 0.00$ & $0.06 / 0.02$ & $18.1 / 0.54$ & $0.61 / 0.20$ & $20.19 / 0.62$ & $0.63 / 0.08$ \\
\hline & prefigured at output & $\mathrm{x}$ & $\mathrm{x}$ & 3 & $0.93 / 0.07$ & $0.14 / 0.01$ & $0.10 / 0.01$ & $0.45 / 0.04$ & $9.45 / 0.44$ & $4.45 / 0.44$ & $10.76 / 0.64$ & $6.35 / 0.68$ \\
\hline
\end{tabular}

* root-sum-squares (RSS) of RMS gradients in horizontal and vertical directions

** $10^{22} \mathrm{~W} / \mathrm{sr}$-TW. Divide by the square of the lens focal length in $\mathrm{cm}$ to obtain irradiance $\left(W / \mathrm{cm}^{2}\right.$ per $\left.\mathrm{TW}\right)$.

Table 2. Results summary of cold system tests at low power 


$$
S=\frac{\left|\int A(x, y) \exp [i \phi(x, y)] d x d y\right|^{2}}{\left|\int A(x, y) d x d y\right|^{2}}
$$

where $A$ is the modeled amplitude and $\phi$ is the measured phase. This formula differs slightly from that of reference [13], in that it singles out and parameterizes the wavefront quality by relating the on-axis intensity in the focal spot to the intensity that would be achieved for a field without phase aberrations, but with the same spatial amplitude profile. The corresponding intensity distributions in the far field were calculated using standard fast Fourier transform techniques and quantified using codes we have developed to analyze Beamlet far-field data. The calculated values for divergence half angle and peak intensity are listed in the table, along with measured values obtained from actual far-field data. Inspection reveals that the measured phase combined with a simple amplitude model closely reproduces the essential features of the measured focal spot; i.e. spot size and peak intensity, lending confidence that the wavefront measurements are accurate. The majority of the entries in the table are shown as two numbers separated by a backslash to indicate the average value obtained from multiple data, and the maximum excursion from the average; the number of data points averaged is also listed.

\section{DISCUSSION}

The output of the Beamlet preamplifier is very close to diffraction limited. The CW measurements show that the AOS improves the wavefront of the preamplifier by $\sim 0.25$ waves to achieve a residual error of 0.32 waves peak to valley, 0.06 waves RMS. In this case the Strehl ratio was 0.87 and the peak far-field intensity was $8.4 \times 10^{22} \mathrm{~W} / \mathrm{sr}-\mathrm{TW}$, which is $81 \%$ of the theoretical maximum for a square beam of this size $\left(P D^{2} / \lambda^{2}\right.$ with $P=1 \mathrm{TW}$ and $\left.D=34 \mathrm{~cm}\right)$. Firing the 5-cm rod added $\sim 0.2$ waves of prompt phase error that was not readily evident unless the AOS was actively correcting the static error. With the rod pumped, wavefront measured with and without the DFM was qualitatively different but similar in peak-to-valley and RMS error. As shown in Figure 2, the $80 \%$ spot size was equivalent for the two cases ( $4.3 \mu \mathrm{rad}$ half angle) but the DFM improved the brightness of the focal spot by $\sim 30 \%$. These results are consistent with those of reference [14].

Beam quality at the output of the system is approximately 2.5 times the diffraction limit with the AOS optimized to correct both prompt and static wavefront errors in the main amplifier. With the preamplifier pumped and the main amplifiers static (rod shot condition), the residual wavefront error at the output of the system was $\sim 1$ wave peak to valley, 0.2 waves RMS, and the $80 \%$ half angle of the focal spot was $10.5 \mu \mathrm{rad}$ (Figure 3a). The measurement was made with the AOS operating closed-loop to maintain a flat wavefront at the output Hartmann sensor up until one second prior to the shot. Data obtained under similar conditions, but with the main amplifiers pumped yielded an output wavefront error of $\sim 3$ waves peak to valley, 0.6 waves RMS, and a much-degraded focal spot (Figure $3 b$ ). The difference between these two wavefronts gives the prompt distortion caused by pumping the large amplifiers, shown in Figure 4 . The phase profile in the horizontal direction is in good agreement with models of propagation through the Beamlet amplifier slabs [15]. The phase profile in the vertical direction shows significant curvature which is not present in the model, and which is not fully understood. Nonetheless, with an appropriate prefigure of the DFM based on this measurement, it was possible to achieve an output wavefront and focal spot on a low-power system shot that were equivalent in quality to the data obtained on rodshots (Figure 3c). In fact, a slight improvement in brightness over the rod-shot condition was observed, which is partly attributed to correction of the small distortion from the $5-\mathrm{cm}$ rod that was included in the measurement of the prompt distortion that we used to establish the prefigure of the DFM.

The above data demonstrates that the 39 -actuator design of the DFM is highly effective at correcting the prompt wavefront distortions incurred in the main amplifier, and that as a result, the focusability of the laser is primarily limited by the static errors in the main amplifier that are not correctable with the AOS. In the case of Beamlet, this residual error has been shown to meet the NIF high-brightness focal spot requirements at both low and high power. Table 3 summarizes the results of high-power focal spot measurements conducted with an optimized AOS at output powers of up to 5.3 TW $(1 \omega)$ and 3.1 TW $(3 \omega)$ in a 200-ps pulse. Amplifier configuration is denoted 11-0 or 11-5, depending on whether the booster amplifier was static or pumped. Maximum power was achieved with the 11-5 configuration, for which the B-integral accumulated in the amplifier was 2.6 radians. The corresponding $80 \%$ power half angles of the $1 \omega$ and $3 \omega$ focal spots were 

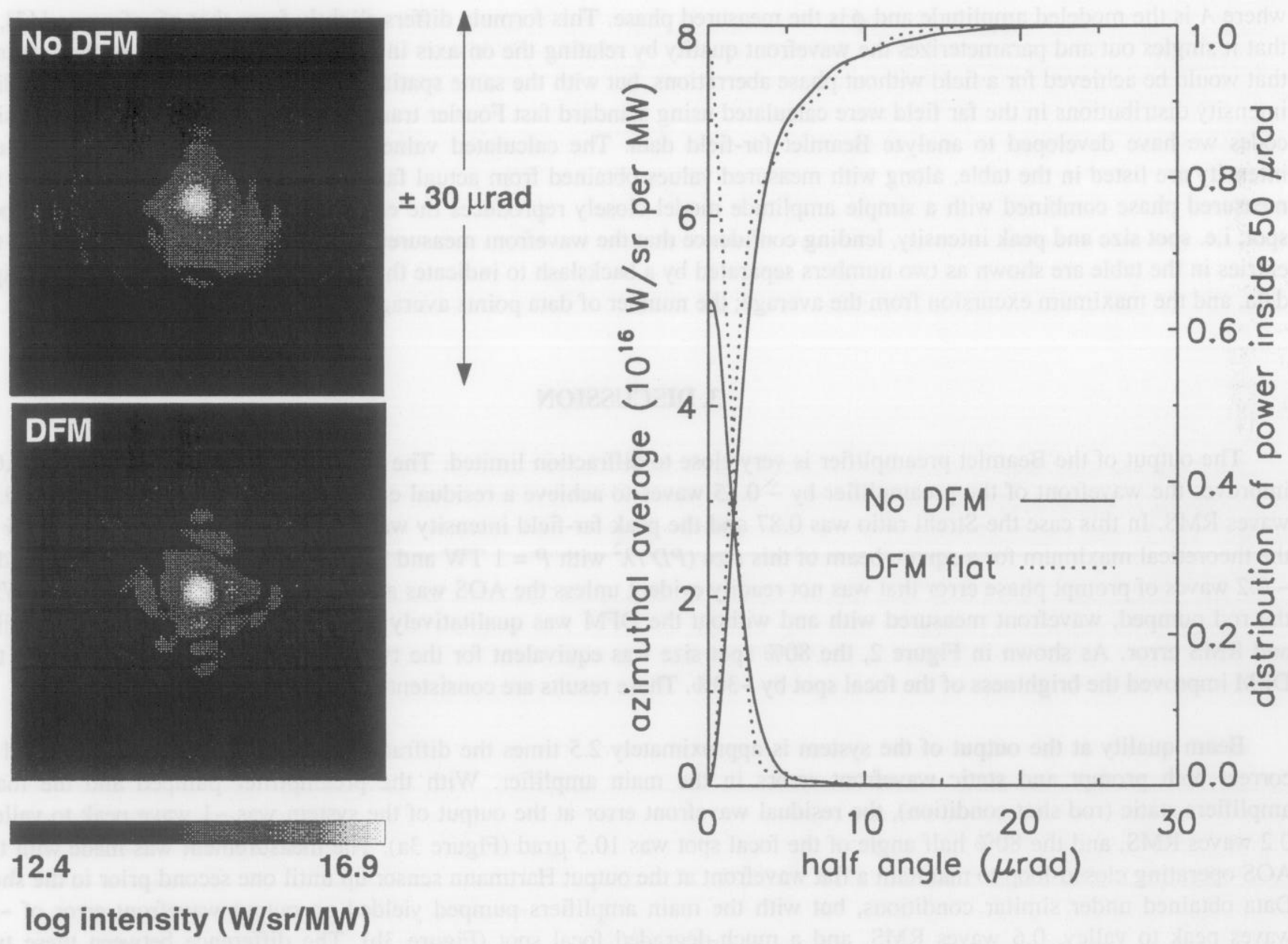

(a)

(b)

Figure 2. (a) Far-field intensity distibutions at the output of the Beamlet preamplifier measured with and without the deformable mirror. Angles are scaled to the main amplifier. (b) Corresponding plots of the azimuthallyaveraged intensity and radial integrations showing the angular distribution of power. In both cases $80 \%$ of the power is contained inside a half-angle of $4.3 \mu \mathrm{rad}$. 


\section{Wavefront}

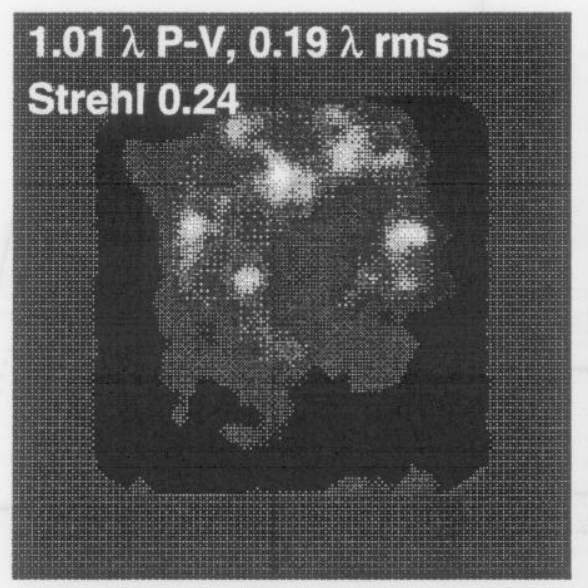

(a)

(b)

(c)
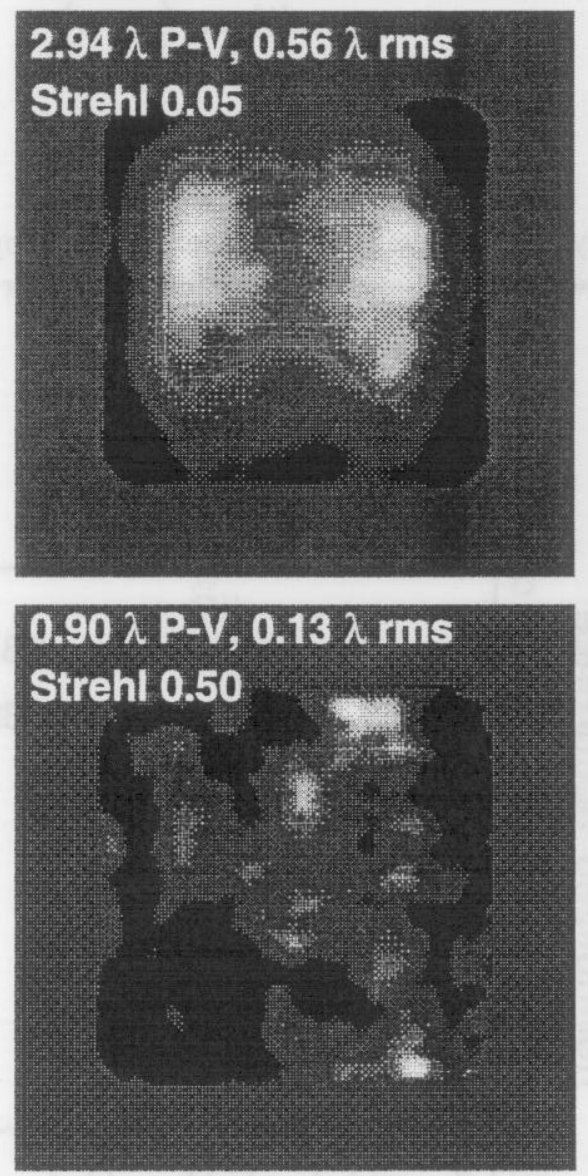

Focal spot
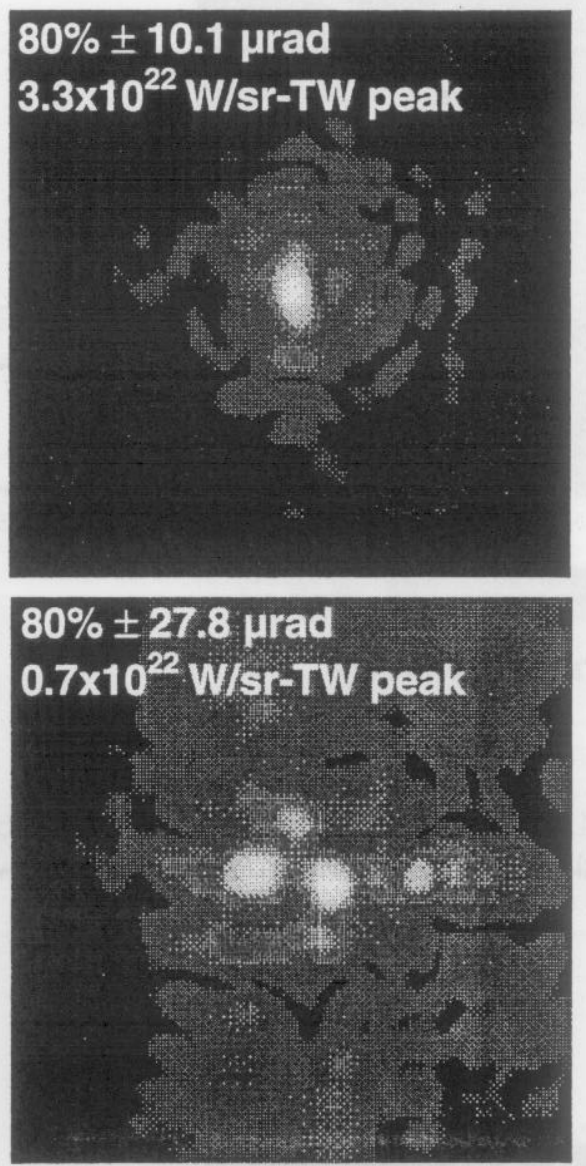

$\pm 30 \mu \mathrm{rad}$

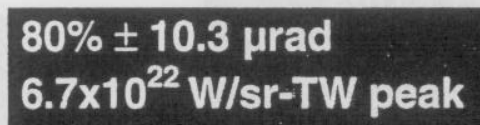

$6.7 \times 10^{22} \mathrm{~W} / \mathrm{sr}-\mathrm{TW}$ peak

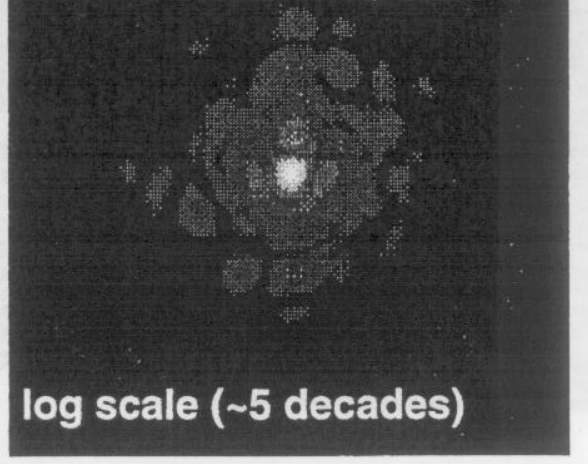

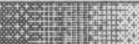

\section{$\min$}

max

Figure 3. $1 \omega$ wavefront and focal spot measured at the output of the main amplifier for (a) rod-shot with the AOS maintaining a flat wavefront up until one second prior to the shot, (b) same AOS condition as (a) but with the main amplifiers pumped, and (c) main amplifiers pumped with the AOS maintaining an optimized prefigured wavefront up until one second prior to the shot. Wavefronts have been weighted by the modeled field amplitude for display. 


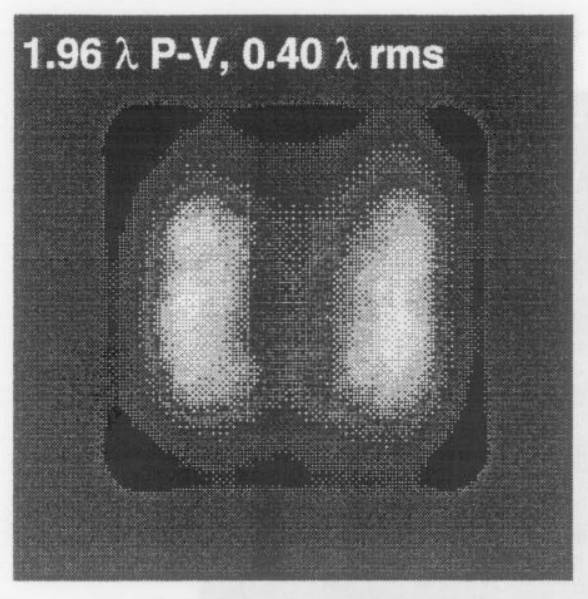

-1.30 phase (waves) 0.66

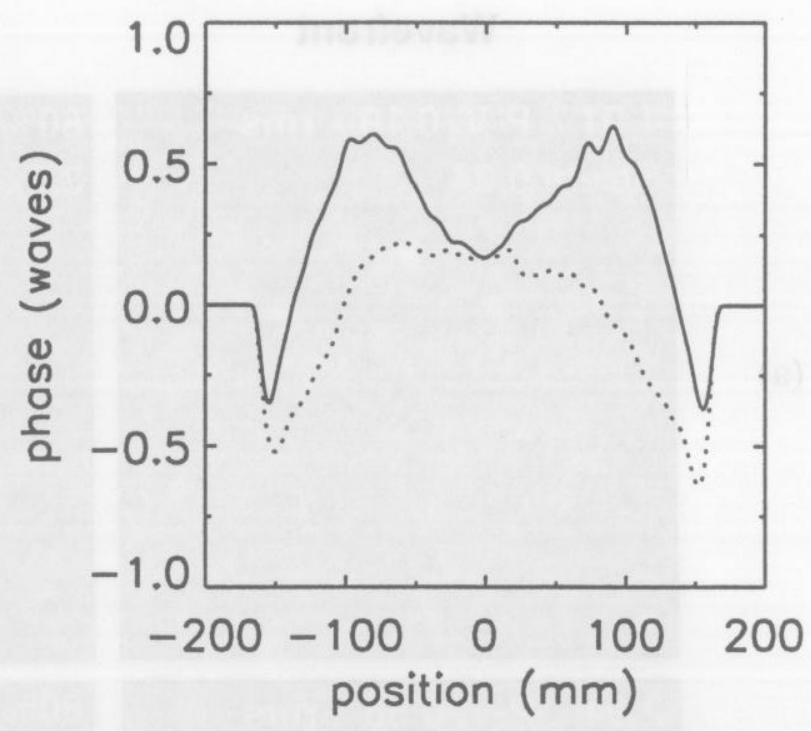

Figure 4. (a) Prompt wavefront distortion caused by pumping the main amplifier, weighted by the modeled field amplitude. (b) Horizontal (__) and vertical (....) linescans through beam center

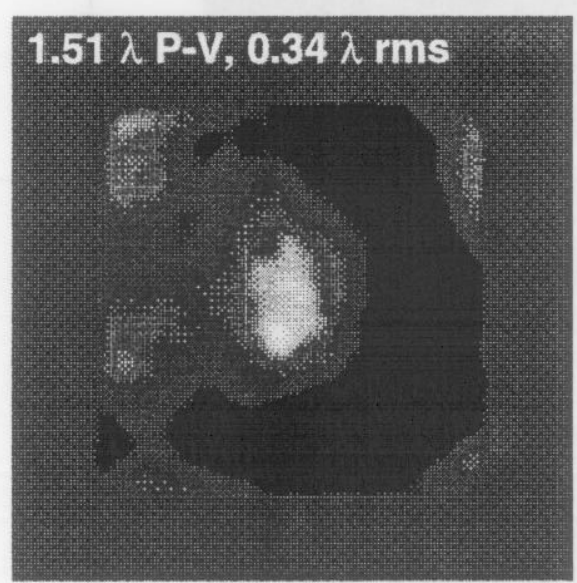

-0.75 phase (waves) $\quad 0.76$

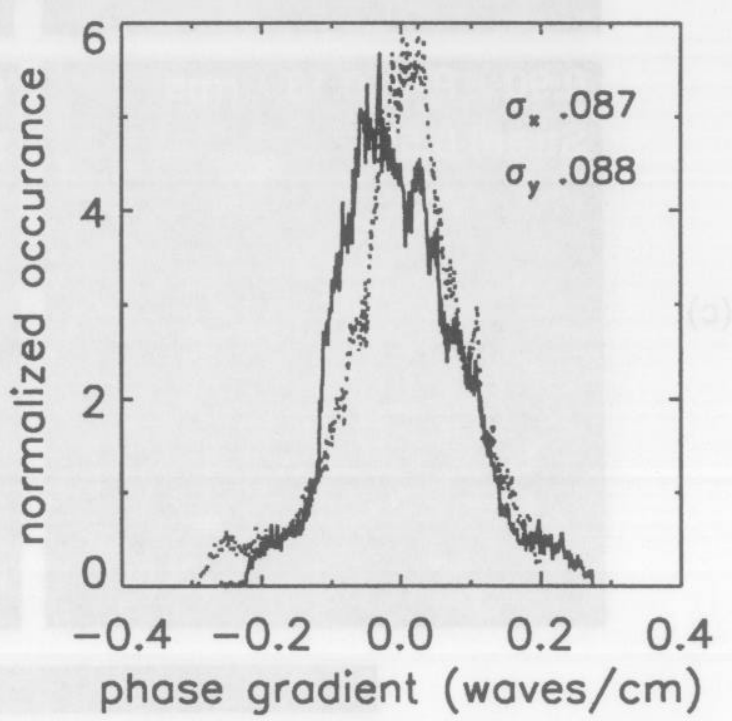

Figure 5. (a) Static wavefront distortion of the main amplifier, weighted by the modeled field amplitude (b) Horizontal (_) and vertical (....) gradient distributions after filtering with a cut-off frequency of $0.03 \mathrm{~mm}^{-1}$. Total RMS gradient is the RSS of $\sigma_{\mathrm{x}}$ and $\sigma_{\mathrm{y}}$. 
12 and $15 \mu \mathrm{rad}$, representing an improvement of approximately $30 \%$ over previous results obtained with a non-optimized AOS [8]. Scaling the power in the $3 \omega$ focal spot $(0.8 \times 3.1 \mathrm{TW})$ by the ratio of NIF to Beamlet beam sizes $\left(1240 \mathrm{~cm}^{2} / 1050 \mathrm{~cm}^{2}\right)$, and multiplying by the number of beams (192) and the transmission of the final optics (0.94) results in a NIF-equivalent performance of $540 \mathrm{TW}$ inside $\pm 15 \mu \mathrm{rad}$. Thus if the quality and associated static errors of the NIF optics are held to Beamlet levels, the NIF focusing requirements should be achievable.

\begin{tabular}{|c|c|c|c|c|c|c|c|c|c|c|}
\hline \multirow[b]{2}{*}{ shot } & \multirow[b]{2}{*}{ amplifier } & \multirow[b]{2}{*}{$\begin{array}{c}\Sigma \mathrm{B}_{1 \omega} \\
\text { (radians) }\end{array}$} & \multirow[b]{2}{*}{$\begin{array}{r}P_{1 \omega} \\
(T W) \\
\end{array}$} & \multirow[b]{2}{*}{$\begin{array}{r}P_{3 \omega} \\
(\mathrm{TW})\end{array}$} & \multicolumn{3}{|c|}{$1 \omega$ focal spot } & \multicolumn{3}{|c|}{$3 \omega$ focal spot } \\
\hline & & & & & $\begin{array}{c}50 \% \\
1 / 2 \text { angle } \\
(\mu \mathrm{rad})\end{array}$ & $\begin{array}{c}80 \% \\
1 / 2 \text { angle } \\
(\mu \mathrm{rad})\end{array}$ & $\begin{array}{c}\text { peak } \\
\text { intensity } \\
*\end{array}$ & \begin{tabular}{|c|}
$50 \%$ \\
$1 / 2$ angle \\
$(\mu \mathrm{rad})$
\end{tabular} & $\begin{array}{c}80 \% \\
1 / 2 \text { angle } \\
(\mu \mathrm{rad})\end{array}$ & \begin{tabular}{|c|} 
peak \\
intensity \\
$*$
\end{tabular} \\
\hline 87082005 & $11-0$ & 1.3 & 1.3 & 0.6 & 5.4 & 11.7 & 3.7 & 8.0 & 13.6 & 0.67 \\
\hline B7082103 & $11-0$ & 2.0 & 2.0 & 1.2 & 5.4 & 11.5 & 3.9 & 7.4 & 12.8 & 0.73 \\
\hline B7082205 & $11-0$ & 2.1 & 2.2 & 1.3 & 5.7 & 11.8 & 3.0 & 8.1 & 13.7 & 0.70 \\
\hline B7082501 & $11-0$ & 2.8 & 2.8 & 1.8 & 5.1 & 11.6 & 3.8 & 8.4 & 14.1 & 0.67 \\
\hline B7082601 & $11-5$ & 1.6 & 3.4 & 2.3 & 5.3 & 12.3 & 3.3 & 7.7 & 12.5 & 0.92 \\
\hline B7082702 & $11-5$ & 1.8 & 3.8 & 2.2 & 4.6 & 11.7 & 3.9 & 6.7 & 12.3 & 0.83 \\
\hline B7082802 & $11-5$ & 2.6 & 5.3 & 3.1 & 4.4 & 12.1 & 4.7 & 6.5 & 14.9 & 0.82 \\
\hline B7082902 & $11-5$ & 2.4 & 5.0 & 3.2 & 4.0 & 11.6 & 4.8 & 7.3 & 15.4 & 0.77 \\
\hline
\end{tabular}

* $10^{22} \mathrm{~W} / \mathrm{sr}-\mathrm{TW}$. Divide by the square of the lens focal length in $\mathrm{cm}$ to obtain irradiance (W/ $\left.\mathrm{cm}^{2}-\mathrm{TW}\right)$

Table 3. Summary of cold system focal spot measurements at high power

The static wavefront errors in the Beamlet amplifier were quantified by calculating the difference between the input and output wavefronts, as measured on a rod shot with a flat mirror in place of the DFM. The result, shown in Figure 5, has proven useful for correlating optics finishing specifications with focal spot performance. For this purpose, it is useful to divide the focal spot into two angular regimes: an outer region, corresponding to divergence angles greater than $\sim 30 \mu \mathrm{rad}$, and an inner region, or central core. Finishing effects of concern for the outer region of the focal spot are high-frequency figure errors, usually associated with polishing, that have characteristic scale lengths less than $\sim 33 \mathrm{~mm}$. The appropriate specification in this regime is the power spectral density of the transmitted wavefront, or PSD [16]. In the central core of the focal spot, corresponding to divergence angles less than $\sim 30 \mu \mathrm{rad}$, the tinishing effects of importance are the longerwavelength figure errors, for which the appropriate specification is the RMS gradient of the transmitted wavefront [17]. Applying a low-pass filter with a cut-off frequency of $0.03 \mathrm{~mm}^{-1}$ to the difference data in the figure, and calculating the RMS gradient of the result yields a value of $1300 \AA / \mathrm{cm}$ for all of the optics combined. Assuming incoherent addition of phase between different elements, and accounting for multiple coherent passes through sections of the amplifier, the average RMS gradient per optic is estimated to be $1300 / 17.2=75 \AA / \mathrm{cm}$. Simulations that use an average gradient distribution based on this result, and nominal PSD's obtained from high-resolution interferograms of individual optics, predict focal spots that are consistent with the Beamlet results [18]. Thus to ensure focal spot performance equivalent to Beamlet, specifications for NIF optics currently limit the RMS gradient of the transmitted wavefront to $70 \AA \mathrm{cm}$ for spatial scale lengths $>33 \mathrm{~mm}$.

\section{CONCLUSION}

Radial shear interferometry has been used to characterize the phase front of the Beamlet prototype laser with high resolution. Test results have shown that the prompt phase errors that result from pumping the 40 -cm aperture slab amplifiers are almost entirely correctable with the Beamlet adaptive optics system, which uses a 39-actuator deformable mirror design that is similar but of smaller scale to what will be fielded on the NIF. Static phase errors in the main amplifier were only partially correctable, resulting in a residual error of $\sim 1$ wave peak to valley, 0.2 waves RMS. The corresponding $1 \omega$ focal spot was approximately 2.5 times the diffraction-limit, with only a small increase in the $80 \%$ power diameter observed for output power levels up to $5.1 \mathrm{TW}$, and total accumulated B-integrals in the main amplifier-of up to 2.6 radians. The corresponding $3 \omega$ focal spot meets the NIF requirement of $500 \mathrm{TW}$ inside of a $250-\mu \mathrm{m}$ diameter circle. 


\section{APPENDIX}

This appendix contains a description of the diagnostics, including the radial shear interferometers, the far-field cameras, and the characterization of their resolution. The layouts for the diagnostics are depicted in Figure 1.

The design of the radial shear interferometers is similar to the three-mirror counter-propagating ring configuration of reference [19]. The beam to be measured enters the interferometer through one of the mirrors which serves as a splitter and is down-collimated by an afocal telescope located inside the ring. The portion of the incident beam that reflects off the splitter travels through the ring in the opposite direction and is up-collimated by the telescope to form the reference beam. The radially sheared beams recombine at the splitter and are relayed to a 1024x1024 pixel CCD camera that records the resulting interference pattern. A high-frequency fringe pattern is essential for the data analysis and is achieved by tilting the splitter. The key difference between this interferometer and the interferometer of reference [19] is our use of the relay properties of the intra-ring telescope to image the splitter onto itself. This feature is critical for measuring the wavefront of the beam at a specific plane, and in addition makes for a stable and alignment-insensitive device.

The first step in extracting wavefront from the radial shear data is accomplished by means of the Fourier transform method of Takeda et al [20]. The high-frequency fringe pattern is first masked with the amplitude model to eliminate background, then Fourier transformed and shifted to move one of the side-band spectrums that is centered at the frequency of the carrier fringes to the center of the grid. At this point the spectrum must be filtered to keep only the shifted side band, and there are many ways to proceed; the method we have chosen is to filter out all frequencies except those lying within a specified pass band about the desired portion of the spectrum. The pass band of the filter establishes the minimum scale length for structure that will be visible in the reconstructed wavefront; for our analysis we used $+/-35 \mu$ rad for the preamplifier data and $+/-70 \mu \mathrm{rad}$ for the output data. Inverse transforming the filtered spectrum yields the complex fringe visibility function, from which the phase (modulo $\pi$ ) is calculated by taking the arctangent of the imaginary part divided by the real part. Unwrapping the $\pi$ transitions in the phase with a straightforward algorithm produces the measured wavefront.

The wavefront reconstructed at this point is not the actual wavefront since the reference wavefront against which it was measured is not planar. To derive the actual wavefront from the data we use an iterative algorithm that starts with a guess that the actual wavefront looks like the wavefront that has been reconstructed

$$
W^{0}=D,
$$

where $W^{0}$ denotes the zeroth-order estimate of the actual wavefront and $D$ represents the processed data. The estimate $W^{0}$ is then used to calculate a new sheared interferogram (i.e. the estimated wavefront minus a radially sheared version of itself), compared with the data, and the difference applied as the first-order correction:

$$
\begin{gathered}
\Delta^{1}=D-\left(W^{0}-W_{\text {sheared }}^{0},\right. \\
W^{1}=W^{0}+\Delta^{1} .
\end{gathered}
$$

A second order correction is then calculated using the first-order wavefront

$$
\begin{gathered}
\Delta^{2}=D-\left(W^{1}-W^{1} \text { sleared }\right), \\
W^{2}=W^{1}+\Delta^{2}
\end{gathered}
$$

and so on until the jth-order estimate of the wavefront reproduces the data with the required accuracy

$$
\Delta^{j}=D-\left(W^{j-1}-W^{j-1} \text { sheared }\right) \approx 0 .
$$

Between iterations the wavefront is also corrected to maintain zero piston. We found this method to converge to an RMS error of $<0.003$ waves between the calculated and measured wavefronts after only three to four iterations.

Irradiance distributions in the far field were recorded with 16-bit scientific grade CCD cameras: a $512 \times 512$ pixel camera located in the front end and a $1024 \times 1024$ pixel camera located in the output sensor. The cameras were measured to 
have magnifications of $0.131 \mu \mathrm{rad} / \mathrm{pixcl}$ for the front-end camera and $0.337 \mu \mathrm{rad} / \mathrm{pixel}$ for the output camera, $\mathrm{referenced} \mathrm{to}$ the plane of the transport spatial filter pinhole. These values are accurate to within $+/-1.8 \%$.

Diagnostic resolution was evaluated using single-mode fiber optic sources in the input and output Hartmann sensor packages that provide reference wavefronts with errors of less than 0.1 waves peak to valley. The reference source in the front end is injected into the beam path near the Hartmann lenslet array and double passed through the sensor optics by means of a mirror inserted near the diagnostic sampling mirror. The resulting wavefront measured with the interferometer has a calculated Strehl of 0.93 and an RMS error of 0.05 waves, most of which is believed to originate in the 6" optics that feed the sensor. In contrast, injecting the reference source directly into the interferometer yields a Strehl of 0.99 and an RMS error of less than 0.02 waves. This small error is ignored, and the measured double-pass wavefront is halved and subtracted from each interferogram to compensate for the sensor optics. This correction is similar to the correction applied to the Hartmann sensor, with the result that both the interferometer and the sensor effectively measure wavefront at the plane of the retro mirror. The resolution of the far field camera was checked by stopping down the reference source to an effective diameter of $340 \mathrm{~mm}$ with a circular aperture and measuring the focal spot. The result showed $80 \%$ of the power inside the diffraction-limited half angle of $3.8 \mu \mathrm{rad}$ (instead of $84 \%$ ), and a measured peak intensity of $7.8 \times 10^{22} \mathrm{~W} / \mathrm{sr}$-TW that is $95 \%$ of the theoretical maximum for this beam size $\left(\mathrm{P}^{2} \mathrm{D}^{2} / 4 \lambda^{2}\right)$. A Strehl ratio of 0.95 inferred from both results is in good agreement with the wavefront measurement. Note that the far field diagnostic is not corrected for the figure of the sensor optics.

A similar calibration was performed for the output diagnostics, except that in this case the mirror for the reference source is located in the 20-mm beam path at the entrance to the sensor, with the result that the large optics that feed the sensor are not sampled. The wavefront of the small optics comprising the sensor is expected to be good and is, having an RMS error of 0.02 waves and a Strehl of 0.98 as measured with the $16 \mathrm{x}$ radial shear interferometer. Measurement of the focal spot with the reference source stopped down to an effective diameter of $325 \mathrm{~mm}$ yielded a peak intensity is $7.5 \times 10^{22}$ W/sr-TW, which is equal to the diffraction limit. To quantify the aberrations of the large optics that feed the sensor we positioned the end of a single-mode fiber at the plane of the transport spatial filter pinhole and measured the transmitted beam in the output sensor. With focus removed, the wavefront error measured with the interferometer was 0.28 waves peak to valley, 0.04 waves RMS, indicating that the focal spots measured in the sensor are representative of conditions in the transport spatial filter.

\section{ACKNOWLEDGMENTS}

We would like to thank the Beamlet Operations crew, who operated the laser and acquired the majority of the data. We also thank John T. Hunt and Erland S. Bliss for several helptul discussions.

*This work was performed under the auspices of the U.S. Department of Energy by Lawrence Livermore National Laboratory under contract No. W-7405-Eng-48.

\section{REFERENCES}

1. J. Murray, J. Auerbach, J. Hunt, K. Manes, R. Sacks, J. Trenholme, and W. Williams, ICF Annual Report, Lawrence Livermore National Laboratory, Livermore, CA, UCRL-LR-105821-97, 99 (1997).

2. W.H. Williams, J.M. Auerbach, M.A. Henesian, J.K. Lawson, P.A. Renard, R.A. Sacks, "The NIF's basic focal spot for temporally flat pulses," paper this proceedings.

3. M.D. Rotter, R.W. McCracken, A.C. Erlandson, and D. Brown, "Thermal recovery measurements on multi-segment amplifiers," Proc. Soc. Photo-Opt. Instrum. Eng., Vol. 2633, 70 (1995).

4. R. Hartley, M. Kartz, W. Behrendt, A. Hines, G. Pollock, E. Bliss, T. Salmon, S. Winters, B. Van Wonterghem, and R. Zacharias, "Wavefront correction for static and dynamic aberrations to within 1 second of the system shot in the NIF Beamlet demonstration facility," Proc. Soc. Photo-Opt. Instrum. Eng., Vol. 3047, 294 (1996).

5. A.C. Erlandson, M.D. Rotter, D.N. Frank, and R.W. McCracken, ICF Quarterly Report, Lawrence Livermore National Laboratory, Livermore, CA, UCRL-LR-105821-95-1, 18 (1994).

6. E.S. Bliss, D.R. Speck, J.F. Holzrichter, J.H. Erkkila, and A.J. Glass, "Propagation of a high-inensity laser pulse with small-scale intensity modulation," Appl. Phys. Lett., Vol. 25, 448 (1974). 
7. J.B. Trenholme, Laser program Annual Report, Lawrence Livermore National Laboratory, Livermore, CA, UCRL50021-75, 237 (1975).

8. P.J. Wegner, C.E. Barker, S.N. Dixit, M.A. Henesian, L.G. Seppala, C.E. Thompson, and B.M. VanWonterghem, "Third-harmonic performance of the Beamlet prototype laser," Proc. Soc. Photo-Opt. Instrum. Eng., Vol. 3047, 370 (1996).

9. B.M. Van Wonterghem, J.R. Murray, J.H. Campbell, D.R. Speck, C.E. Barker, I.C. Smith, D.F. Browning, and W.C. Behrendt, "Performance of a prototype for a large-aperture multipass Nd:glass laser for inertial confinement fusion," Appl. Opt. Vol. 36, 4932 (1997).

10. J.T. Salmon, E.S. Bliss, J.L. Byrd, M. Feldman, M.A. Kartz, J.S. Toeppen, B. Vam Wonterghem, and S.E. Winters, "An adaptive optics system for solid state lasers used in inertial confinement fusion," Proc. Soc. Photo-Opt. Instrum. Eng., Vol. 2633, 105 (1995).

11. B.M. Van Wonterghem, J.T. Salmon, and R.W. Wilcox, ICF Quarterly Report, Lawrence Livermore National Laboratory, Livermore, CA, UCRL-LR-105821-95-1, 42 (1994).

12. C. La Fiandra, P. Mehta, G. Goldstein, R. A. Zacharias, and S. Winters, "NIF deformable mirror," paper this proceedings.

13. M. Borne and E. Wolfe, Principles of Optics, Pergamon Press, New York, 1991.

14. B. M. Van Wonterghem, D. R. Speck, et al, "A compact and versatile pulse generation and shaping subsystem for high energy laser systems," Proc. Soc. Photo-Opt. Instrum. Eng., Vol. 1870, 64 (1993).

15. M.D. Rotter, private communication, Lawrence Livermore National Laboratory, Livermore, CA (1998).

16. J.K. Lawson, D.M. Aikens, R.E. English Jr., and C.R. Wolfe, "Power spectral density specifications for high-power laser systems," Proc. Soc. Photo-Opt. Instrum. Eng., Vol. 2775, 345 (1996).

17. J.K. Lawson, J.M. Auerbach, R.E. English Jr., M.A. Henesian, J.T. Hunt, R.A. Sacks, J.B. Trenholme, W.H. Williams, M.J. Shoup III, J.H. Kelly, and C.T. Cotton, "NIF optical specifications- the importance of the RMS gradient," paper this proceedings.

18. W.H. Williams, J.M. Auerbach, M.A. Henesian, J.K. Lawson, J.T. Hunt, R.A. Sacks, and C. C. Widmayer, "Modeling characterization of the National Ignition Facility focal spot," presented at LASE '98, San Jose, CA (1998).

19. M.V.R.K. Murty,"A compact radial shearing interferometer based on the law of refraction," Appl. Opt., Vol. 3, 853 (1964).

20. M. Takeda, H. Ina, and S. Kobayashi, "Fourier-transform method of fringe-pattern analysis for computer-based topography and interferometry," JOSA, Vol. 72, 156 (1981). 